\title{
The underlying model for defect parameters in materials of geophysical interest and Seismic Electric Signals
}

\author{
E. Dologlou \\ Solid State Section, Department of Physics, University of Athens, Panepistimiopolis, Zografos 15784 Athens, Greece \\ Correspondence to: E. Dologlou (edologl@phys.uoa.gr)
}

Received: 5 October 2011 - Accepted: 2 November 2011 - Published: 6 March 2012

\begin{abstract}
Here, we investigate whether the bulk or the shear is the appropriate modulus for the defect parameters in different materials by focusing on those of geophysical interest. We show that the self-diffusion process and just also the bulk modulus should be related to defect parameters. It is this interrelation which accounts for the emission of electric signals before fracture, and is thus in accordance with the physical basis of the Seismic Electric Signal prior to earthquakes in Greece.
\end{abstract}

\section{Introduction}

During the last 3 decades, low frequency $(\leq 1 \mathrm{~Hz})$ transient changes of the Earth's electric field, termed Seismic Electric Signals (SES), have been found to precede major earthquakes in Greece (Varotsos and Alexopoulos, 1984a, b; Varotsos et al 1986, 1988, 1993b ). The model proposed by Varotsos and Alexopoulos, 1986 for the SES generation is based on the pressure stimulated polarization currents (PSPC) which are emitted from a solid containing electric dipoles upon a gradual increase of the pressure $P$. These dipoles, which are formed between aliovalent impurities and vacancies that appear in the crystal for charge compensation (Kostopoulos et al., 1975), change their orientation with a relaxation time $\tau$ given as $\tau=(\lambda v)^{-1} \exp (g / k T)$, where $v$ is the attempt frequency for a jump to a number of $\lambda$ accessible paths, $T$ is the temperature and $g$ is the Gibbs energy for the re-orientation process (activation). Pressure affects the value of $g$ according to the relation $v=(d g / d P)_{T}$ where $v$ stands for the migration or activation volume. In case that $v<0$ (Varotsos and Alexopoulos, 1980b; Varotsos et al., 1993a) an increase in pressure leads to a decrease of the relaxation time $\tau$. When the increasing pressure reaches a critical value $P=P_{\mathrm{cr}}$, a transient electric current arising from the cooperative re orientation of dipoles is emitted. This transient current constitutes the SES (Varotsos and Alexopoulos, 1984a, b).
Zhang et al. (2011) investigated the temperature and pressure dependence of oxygen self-diffusion coefficients (see below) in a variety of materials of geophysical interest such as $\mathrm{Mg}_{2} \mathrm{SiO}_{4}$ polymorfs (forsterite, wadsleyite and ringwoodite) and $\mathrm{MgSiO}_{3}$ perovskite. They found that the activation energy as well as the activation volume are directly related to the bulk modulus through the $c B \Omega$ model (Varotsos and Alexopoulos, 1986), of which a brief description will be given below. A similar relation of the defect Gibbs activation energy and activation volume to the bulk modulus has been also verified by Dologlou (2010), in the case of self diffusion in lithium hydride.

Here, we check whether in various defect processes the shear or the bulk is the appropriate elastic modulus for the interconnection of the defect parameters with the bulk properties focusing on materials prevailing in the Earth's mantle and thus of geophysical interest.

\section{The $c B \Omega$ model}

Defect parameters can be directly estimated by means of the $c B \Omega$ model which interconnects the Gibbs energy $g^{i}$ with the bulk expansivity and elastic data according to the formula (Varotsos, 1976, 1977, 2007a; Varotsos and Alexopoulos, 1977, 1978, 1980a, 1986; Varotsos et al., 1978):

$g^{i}=c^{i} B \Omega$

where the superscript " $i$ " stands for the different process mechanism, (formation, migration and activation), $B$ is the isothermal bulk modulus, $\Omega$ is the mean atomic volume, and $c^{i}$ is a dimensionless constant which can be considered as independent of temperature and pressure. This model has been successfully applied to a variety of cases including rare gas solids (Varotsos and Alexopoulos, 1984c), alkali and silver halides (Varotsos and Alexopoulos, 1978, 1979, 1986; Varotsos and Miliotis, 1974; Kostopoulos et al., 1975), alkali halide mixed crystals (Varotsos and Alexopoulos, 1980c; 
Varotsos, 1981), diamond (Varotsos, 2007b), fluorine superionic semiconductors (Varotsos, 1976; 2008), as well as for the electric signals emitted from crystalline materials under pressure in a similar fashion as in the case of seismic electric signals (SES) detected prior to large earthquakes (Varotsos and Alexopoulos, 1984a, b; Varotsos and Lazaridou, 1991; Varotsos et al., 2002, 2005, 2006a, b, 1986).

\section{Application of the $c B \Omega$ model in periclase}

For a single operating mechanism, the self-diffusion process at different temperatures $T$, is described in terms of activation Gibbs energy $g^{\text {act }}$ as

$D=f \alpha^{2} v_{D} \exp \left(-g^{\text {act }} / k_{B} T\right)$

where $f$ is a numerical constant depending on the diffusion mechanism and the structure, $\alpha$ the lattice constant and $v$ is the attempt frequency. A combination of Eqs. (1) and (2) leads to

$D=f \alpha^{2} v_{D} \exp \left(-c^{\text {act }} B \Omega / k_{B} T\right)$

If for constant temperature $T$ (or pressure $P$ ) and for a given pressure $P$ (or temperature $T$ ) the self diffusion coefficient $D_{i}$ is known, the constant $c^{\text {act }}$ can be estimated from the formula:

$c^{\text {act }}=-\frac{k T}{B_{i} \Omega_{i}} \ln \frac{D_{i}}{f \alpha_{i}^{2} v_{D}}$

where the subscript " $i$ " stands for the value of each parameter at $P=P_{i}$ (or $T=T_{i}$ ).

Once the $c^{\text {act }}$ has been calculated, the self diffusion coefficient $D$ at any other pressure and/or temperature can be derived by using Eq. (3) if the values of $\alpha, v_{D}, \mathrm{~B}$ and $\Omega$ at each pressure and/or temperature are known.

We will now proceed to the estimation of the self diffusion coefficient of oxygen in periclase $(\mathrm{MgO})$ at temperature $T=$ $2000 \mathrm{~K}$ and pressure range (9.21-117) GPa for which experimental data are published (Ita and Cohen, 1997). These are temperature and pressure conditions similar to those prevailing in the lower mantle. Periclase is one of the major earthforming minerals with a NaCl-type structure and it plays a significant role in the physics and chemistry of the lower mantle of the Earth and in related seismology and geodynamics.

At room pressure and room temperature, the lattice constant is $\alpha_{0}=4.20 \times 10^{-8} \mathrm{~cm}$ (Geneste et al., 2009) and consequently the atomic volume $\Omega_{0}=\alpha^{3} / 8=9.261 \times 10^{-24} \mathrm{~cm}^{3}$. The values of the bulk modulus $B$ at $T=2000 \mathrm{~K}$ and for the pressure range (9-117) GPa are taken from elastic and expansivity data reported by Sushil (2005) (Table 6). The Debye temperature is $\Theta_{D}=927.4 \mathrm{~K}$ (Zhao et al., 2007) and thus $v_{D}=19.315 \times 10^{12} \mathrm{~s}^{-1}$ while the numerical constant is taken $f=0.78$.

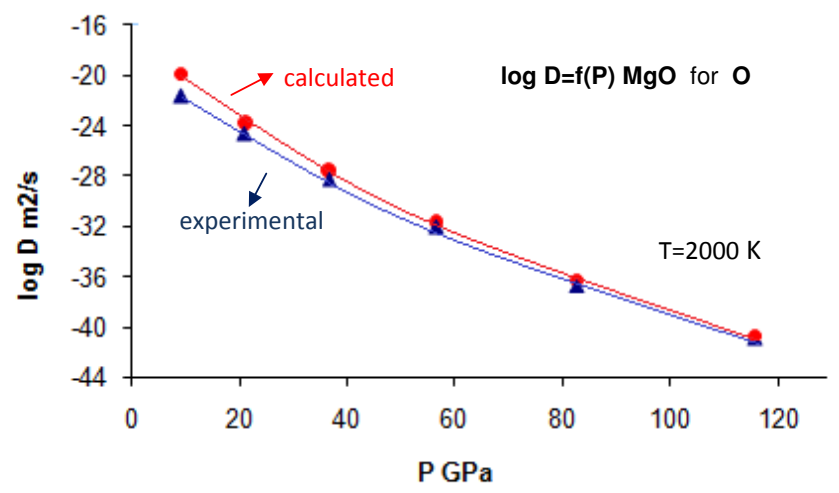

Fig. 1. Pressure dependence of the self diffusion coefficient of $O$ in $\mathrm{MgO}$ for $T=2000 \mathrm{~K}$. Triangles denote the experimental data and solid circles the calculated ones.

Based on the elastic and expansivity data of Sushil (2005) (Tables 3 and 4), we calculate the values of $\alpha$ and $\Omega$ for different pressures at $T=2000 \mathrm{~K}$. Let us give an example for $P=117 \mathrm{GPa}$. From Table 3 of Sushil (2005), $V(T, P) / V\left(T_{0}, 0\right)=\Omega(2000,0) / \Omega\left(T_{0}, 0\right)=1.0798$ and thus $\Omega(2000,0)=1.0798 \times 9.261 \times 10^{-24} \mathrm{~cm}^{3}=10.00003 \times$ $10^{-24} \mathrm{~cm}^{3}$. On the other hand from Table 4. of Sushil (2005), $V(T, P) / V(T, 0)=\Omega(2000,117) / \Omega(2000,0)=0.6609$ which leads to $\Omega_{(2000,117)}=6.6091 \times 10^{-24} \mathrm{~cm}^{3}$ and thus $\alpha_{(2000,117)}=3.7532 \times 10^{-8} \mathrm{~cm}$.

By inserting in Eq. (4) for the highest pressure $P=117 \mathrm{Gpa}$, the corresponding values $\alpha_{(2000,117)}=$ $3.7532 \times 10^{-8} \mathrm{~cm}, \Omega_{(2000,117)}=6.6091 \times 10^{-24} \mathrm{~cm}^{3}, B=$ 5160 kbars (Sushil, 2005; Table 6) and the diffusion coefficient $D=4.03 \times 10^{-42} \mathrm{~m}^{2} \mathrm{~s}^{-1}$ for oxygen (Ita and Cohen, 1997) we obtain $c^{\text {act }}=0.666 \pm 0.01$ (the error is due to the uncertainty in the estimation of $B$ and $v_{D}$ ).

Through Eq. (3), the diffusion coefficients $D$ of oxygen are estimated for the pressure range (9-117) GPa for which experimental data are available (Ita and Cohen, 1997). The derived relation $\log (D)=f(P)$ is shown in Fig. 1 where circles denote the calculated diffusion values and triangles the experimental ones (Ita and Cohen, 1997).

Thus, from a single measurement and by means of the $c B \Omega$ model, the diffusion coefficients can be successfully reproduced in a number of representative earth-forming materials and crystalline solids (Zhang et al., 2011; Varotsos and Alexopoulos, 1986; Dologlou, 2010).

We will now examine whether the bulk or the shear modulus is the appropriate model for those materials. The defect volume $v$ is defined as:

$v=(d g / d P)$

By inserting Eq. (1) $(g=c B \Omega)$ in Eq. (5) we have:

$v / g=(d B / d P-1) / B$

Since the quantity $(d B / d P)_{T}$ significantly exceeds unity, e.g. usually $(d B / d P)_{T}$ lies in the range 4 to 8 , the above 
Eq. (6) shows that $v$ is positive, thus implying that $D$ decreases with increasing pressure, which is in agreement with experimental data.

On the other hand, if we consider that $g$ is proportional to the shear modulus $C$ (Zener, 1951) we find that

$v^{\mathrm{m}} / g^{\mathrm{m}}=-1 / B+1 / C(d C / d P)$

where $v^{\mathrm{m}}$ and $g^{\mathrm{m}}$ the migration volume and energy, respectively. In a later work Flynn (1992) used another combination of elastic constants instead of the shear modulus.

Let us now consider the case of simple crystalline solids, such as silver halides (i.e. $\mathrm{AgBr}, \mathrm{AgCl}$ ) which provide an excellent example of the dependence of activation volume and activation Gibbs energy on the bulk modulus (Varotsos and Alexopoulos, 1986). In silver halides, the pressure derivative of shear modulus is negative, i.e. $d C / d P<0$ (Cain, 1977). In that case, from Eq. (7) it is obvious that the migration volume $v^{\mathrm{m}}$ becomes negative and thus, $D$ increases with increasing pressure which contradicts the experimental facts.

From the above analysis, it seems that the defect parameters are directly related to the bulk modulus for materials of geophysical interest and crystalline solids.

\section{Conclusions}

Here, we have checked whether the bulk or shear modulus is related to defect parameters focusing on materials of geophysical interest. With the aid of the $c B \Omega$ thermodynamical model, in a variety of such materials as $\mathrm{Mg}_{2} \mathrm{SiO}_{4}$ polymorfs, perovskite and periclase, as well in crystalline solids, the activation Gibbs energy and the activation volume are directly related to the bulk modulus. As a first application, from a single measurement we successfully reproduced by means of the aforementioned model the self diffusion coefficients of oxygen in periclase for the pressure range (9-117) GPa at $T=2000 \mathrm{~K}$, which are the conditions dominating in the lower Earth's mantle. As a second application, we calculated the defect activation volumes in silver halides and we found that the bulk modulus leads to a positive value for the defect activation volume while the shear modulus to a negative one which contradicts experimental facts.

Thus, materials prevailing in the lower mantle obey the $c B \Omega$ model which has been also found to describe satisfactorily the parameters of the electric signals preceding fracture and hence the SES prior to earthquakes.

Edited by: M. E. Contadakis

Reviewed by: two anonymous referees

\section{References}

Cain, L. S.: The elastic constants and their temperature and pressure derivatives, J. Phys. Chem. Solids, 38, 73-79, 1977.

Dologlou, E.: Hydrogen and deuterium diffusion in lithium hydride, J. Appl. Phys., 107, 083507, doi:10.1063/1.3387861, 2010.

Flynn, C. P.: Point defects and Diffusion, Clarendon Press-Oxford, 1972.

Geneste, G., Hayoun, M., Finocchi, F., and Morillo, J.: Competing mechanisms in the atomic diffusion of a $\mathrm{MgO}$ admolecule on the $\mathrm{MgO}(001)$ surface, J. Phys. Condens. Matter, 21, 315004315010, 2009.

Ita, J. and Cohen, R. E.: Effects of Pressure on Diffusion and Vacancy Formation in $\mathrm{MgO}$ from Nonempirical Free-Energy Integrations, Phys. Rev. Lett., 79, 3198-3201, 1997.

Kostopoulos, D., Varotsos, P., and Mourikis, S.: Conductivity of crystalline NaI, Can. J. Phys., 53, 1318-1320, 1975.

Sushil, K.: Volume dependence of isothermal bulk modulus and thermal expansivity of MgO, Physica B , 367, 114-123, 2005.

Varotsos, P.: Comments on the formation entropy of a Frenkel defect in $\mathrm{BaF}_{2}$ and $\mathrm{CaF}_{2}$, Phys. Rev. B, 13, p. 938, 1976.

Varotsos, P.: On Temperature and pressure dependence of defect formation volume in ionic crystals, J. Phys. (France), 38, L455L458, 1977.

Varotsos, P.: Determination of the composition of the maximum conductivity or diffusivity in mixed alkali halides, J. Phys. Chem. Solids, 42, 405-407, 1981.

Varotsos, P.: Comparison of models that interconnect point defect parameters, J. Appl. Phys., 101, 123503, doi:10.1063/1.2745359, 2007a.

Varotsos, P.: Calculation of point defect parameters in diamond, Phys. Rev. B, 75, 172107, p. 3, doi:10.1103/PhysRevB.75.172107, 2007b.

Varotsos, P.: Point defect parameters in b-PbF2 revisited, Solid State Ionics, 179, 438-441, 2008.

Varotsos, P. and Alexopoulos, K.: Estimation of the migration enthalpy and entropy for cation vacancy motion in alkali halides with the NaCl-type structure, Phys. Rev. B, 15, 2348-2351, 1977.

Varotsos, P. and Alexopoulos, K.: The curvature in conductivity plots of silver halides as a consequence of anharmonicity, J. Phys. Chem. Solids, 39, 759-761, 1978.

Varotsos, P. and Alexopoulos, K.: On the possibility of the enthalpy of a Schottky defect decreasing with increasing temperature, J. Phys. C Solid State, 12, L761-L764, 1979.

Varotsos, P. and Alexopoulos, K.: Calculation of Diffusion Coefficients at Any Temperature and Pressure from a Single Measurement: I. Self-Diffusion, Phys. Rev. B, 22, 3130-3134, 1980a.

Varotsos, P. and Alexopoulos, K.: Negative activation volumes of defects in solids, Phys. Rev. B, 21, 4898-4899, 1980b.

Varotsos, P. and Alexopoulos, K.: Prediction of the Compressibility of Mixed Alkali Halides, J. Phys. Chem. Solids, 41, 1291-1294, 1980c.

Varotsos, P. and Alexopoulos, K.: Physical properties of the variations of the electric field of the earth preceding earthquakes, I, Tectonophysics, 110, 73-98, 1984a.

Varotsos, P. and Alexopoulos, K.: Physical properties of the variations of the electric field of the earth preceding earthquakes, II. Determination of epicentre and magnitude, Tectonophysics, 110, 99-125, 1984b. 
Varotsos, P. and Alexopoulos, K.: Connection between the formation volume and formation Gibbs energy in noble gas solids, Phys. Rev. B, 30, 7305-7306, 1984c.

Varotsos, P. and Alexopoulos, K.: Thermodynamics of Point Defects and their Relation with the Bulk Properties, North Holland, Amsterdam, 1986.

Varotsos, P. and Lazaridou, M.: Latest aspects of earthquake prediction in Greece based on seismic electric signals. I, Tectonophysics, 188, 321-347, 1991.

Varotsos, P. and Miliotis, D.: New Aspects on the Dielectric Properties of the Alkali Halides with Divalent Impurities, J. Phys. Chem. Solids, 35, 927-930, 1974.

Varotsos, P., Ludwig, W., and Alexopoulos, K.: Calculation of the Formation Volume of Vacancies in Solids, Phys. Rev. B, 18, 2683-2691, 1978.

Varotsos, P., Alexopoulos, K., Nomicos, K., and Lazaridou, M.: Earthquake prediction and electric signals, Nature, 322, p. 120, 1986.

Varotsos, P., Alexopoulos, K., Nomicos, K., and Lazaridou, M.: Official Earthquake prediction procedure in Greece, Tectonophysics, 152, 193-196, 1988.

Varotsos, P., Alexopoulos, K., and Lazaridou, M.: Latest aspects of earthquake earthquake prediction in Greece based on Seismic Electric Signals II, Tectonophysics, 224, 1-37, 1993a.
Varotsos, P., Alexopoulos, K., Lazaridou, M., and Nagao, T.: Earthquake predictions issued in Greece by seismic electric signals since February 6, 1990, Tectonophysics, 244, 269-288, 1993 b.

Varotsos, P. A., Sarlis, N. V., and Skordas, E. S.: Long-range correlations in the electric signals that precede rupture, Phys. Rev. E, 66, 011902, doi:10.1103/PhysRevE.66.011902, 2002.

Varotsos, P. A., Sarlis, N. V., Skordas, E. S., and Lazaridou, M.: Natural entropy fluctuations discriminate similar looking electric signals emitted from systems of different dynamics, Phys. Rev. E, 71, 011110, doi:10.1103/PhysRevE.71.011110, 2005.

Varotsos, P., Sarlis, N., Skordas, E., Tanaka, H., and Lazaridou, M., Entropy of seismic electric signals: analysis in natural time under time reversal, Phys. Rev. E, 73, 031114, doi:10.1103/PhysRevE.73.031114, 2006a.

Varotsos, P., Sarlis, N., Skordas, E., Tanaka, H., and Lazaridou, M.: Attempt to distinguish long-range temporal correlations from the statistics of the increments by natural time analysis, Phys. Rev. E, 74, 021123, doi:10.1103/PhysRevE.74.021123, 2006b.

Zener, C.: Theory of Do for Atomic Diffusion in Metals, J. Appl. Phys., 22, 372-375, 1951.

Zhang, B., Wu, X., and Zhou, R.: Calculation of oxygen selfdiffusion in $\mathrm{Mg}_{2} \mathrm{SiO}_{4}$ polymorphs and $\mathrm{MgSiO}_{3}$ perovskite based on the compensation law, Solid State Ionics, 186, 20-28, 2011.

Zhao, J. Z., Lu, L. Y, Chen, X. R., and Bai, Y. L.: First-principles calculations for elastic properties of the rocksalt structure $\mathrm{MgO}$, Physica B, 387, 245-249, 2007. 